\title{
ESTIMATION OF DYADIC CHARACTERISTICS OF FAMILY NETWORKS USING SAMPLE SURVEY DATA
}

\author{
By Chris Skinner ${ }^{\ddagger *, \dagger}$ AND Fiona Steele ${ }^{\ddagger, \dagger}$ \\ London School of Economics \& Political Science \& $^{\ddagger}$
}

\begin{abstract}
We consider the use of sample survey data to estimate dyadic characteristics of family networks, with an application to non-coresident parent-child dyads. We suppose that survey respondents report either from a parent or child perspective about a dyad, depending on their membership of the dyad. We construct separate estimators of common dyadic characteristics using data from both a parent and a child perspective and show how comparisons of these estimators can shed light on data quality issues including differential missingness and reporting error. In our application we find that a simple missingness model explains some striking patterns of discrepancies between the estimators and consider the use of poststratification and a related redefinition of count variables to adjust for these discrepancies. We also develop approaches to combining the separate estimators efficiently to estimate means and frequency distributions within subpopulations.
\end{abstract}

1. Introduction. Many social science research questions are framed in terms of family networks. For example, how does parents' provision of financial support and grandchild care to their adult children depend on parents' health and socioeconomic status (e.g. Henretta, Grundy and Harris, 2002)? How does the level and nature of support given by individuals to their elderly parents vary across countries (e.g. Brandt, Haberkern and Szydlik, 2009)? Widmer, Aeby and Sapin (2013) give other examples of why it is increasingly important in research on contemporary family networks to look beyond household boundaries. Obtaining statistical evidence from sample surveys to address such questions can be challenging, however. Whereas the practice in household surveys of obtaining data from different family members who live together is well established, it is less straightforward to obtain data from family network members who are non-coresident, that is live in different locations.

\footnotetext{
${ }^{*}$ Corresponding author.

${ }^{\dagger}$ Supported by a UK Economic and Social Research Council (ESRC) grant "Methods for the Analysis of Longitudinal Dyadic Data with an Application to Inter-generational Exchanges of Family Support" (ref. ES/P000118/1).

MSC 2010 subject classifications: Primary 60K35, 60K35; secondary 60K35

Keywords and phrases: coresident, network sampling, poststratification, reporting error
} 
At least three large-scale survey designs are used in practice to study noncoresident members of family networks. In one, individual respondents in a 'main' survey are asked for their consent to approach family network members living elsewhere and a follow-up survey is then undertaken. Shapiro (2004) and Mandemakers and Dykstra (2008) describe examples in the United States and the Netherlands. A second design is a panel/longitudinal survey in which family members, living together at the start of the survey in sample households, are each followed up for many years, sufficient for sample family members living apart to provide suitable data on non-coresident family networks. Altonji, Hayashi and Kotlikoff (1997) give a United States example with networks formed after children have left their parental home. Both designs have limitations. The first is costly and the need for consent of both the main sample member and the non-coresident relative may lead to a selective sample of pairs of network members, for example those with a good relationship. The second design can also lead to a selective sample since it requires that the family members lived together during the panel observation period. In this paper we focus on a third design which seems more widely used, simpler and less expensive. In this approach, no attempt is made to collect data from non-coresident individuals directly. Instead, individual respondents in a standard individual or household survey are asked questions, effectively by proxy, about family network members living elsewhere. This design is employed in the family networks module included in the British Household Panel Survey (BHPS) and its successor Understanding Society, and data from the 2001 wave of BHPS will provide the basis for the application in this paper. Similar data are available in other household panel surveys, for example the US Panel Survey of Income Dynamics, and the cross-national Gender and Generations Programme.

As with any data collection by proxy, it is important to consider issues of data quality, such as missing data or measurement error. In this paper, we shall develop a new approach to assessing data quality for the third form of survey design. We shall focus on dyadic aspects of family networks, where a dyad refers to an ordered pair of members of a network and in our application consists of two non-coresident adult individuals where one is a parent of the other. Any individual survey respondent may belong to multiple dyads and may be a parent in some and a child in others. We suppose that respondents are asked to report about each of these dyads, from either a parental or child perspective, depending on the nature of their membership of the dyad. This generates two sources of data about dyads, which we refer to as the parent data and the child data.

We consider the estimation of dyadic characteristics, such as the number 
of dyads of a certain type in the network. It is possible to construct a single estimator of such a parameter employing all the data by treating the design as a form of network sampling (Lohr, 2010) and using, for example, Horvitz-Thompson estimation (Frank, 1977). In this paper, we consider instead constructing separate estimators of such a parameter using the parent and child data in turn. The aim is for comparisons of these two estimators to shed light on possible sources of reporting error or missing data which may lead to bias. The first and main part of this paper explores the use of such comparisons for data quality assessment, not only as a diagnostic method but also to suggest adjustments to the estimation methodology, including weighting and a related redefinition of variables. Later, we develop novel methods for combining separate estimators to provide more efficient estimation.

We shall focus in this paper on basic demographic dyadic characteristics, defined in terms of age and sex, although our approach is, in principle, applicable more broadly. We take the characteristics to be finite population parameters, as might be of interest for descriptive purposes in official statistics. We assume that the sampling scheme is such that the number of dyads for which both parent and child data are available is negligible so that it is not possible to use such matched data to assess the concordance of reports, as may be feasible in either of the first two survey designs (see Section 2).

The rest of this paper is organised as follows. Section 2 sets out some related literature, primarily on issues of data quality for dyadic data on families. Section 3 sets out the basic methodological framework, introducing separate estimators based on parent and child data. The data we use from the BHPS and the application are described in Section 4. Section 5 provides our first comparison of estimates obtained from applying the estimators introduced in Section 3 to the BHPS data. This analysis suggests that discrepancies between the estimates are attributable to differential missingness and the use of weighting to correct for differential missingess is discussed in Section 6. Related issues of reporting error are discussed in Section 7. The efficient combination of estimators based on parent and child data is discussed in Section 8. Section 9 concludes with a discussion of extensions.

2. Related literature. The estimation of dyadic characteristics is an established problem in the network sampling literature (e.g. Frank, 1977) and likelihood methods for inference in the presence of missing data have been considered by Handcock and Gile (2007, 2010). However, we are not aware that the approach proposed here for assessing data quality by comparing separate estimates has been investigated systematically before. There 
is some relevant applied literature which compares reports of subsamples of parent and child (or mother and father) survey respondents regarding the same kind of dyadic phenomena.

Chan and Ermisch (2011) use data from the BHPS family networks module, as in our study, to assess consistency of parent and child reports of intergenerational exchanges of different types of support. For example, child reports of help given to non-coresident parents were compared with parent reports of help received from non-coresident adult children, based on unmatched subsamples of parent and child respondents. They concluded that "the overall picture of help given by children is comparable to help received by parents, and vice versa". Studies employing the first design described in Section 1 use matched data on parent-child dyads to assess the concordance of parent and child reports of variables such as frequency of contact, exchanges of support, and relationship quality (Shapiro, 2004; Mandemakers and Dykstra, 2008), rather than the more basic demographic variables considered here. The focus of these studies is to identify individual determinants of discrepancies in reports, which are attributed to a number of sources including differences in perceptions and inaccuracies in reporting (Mandemakers and Dykstra, 2008). There is some discussion of other data quality issues, such as nonresponse, in these papers. However, the nature of nonresponse and other kinds of sample selection is likely to be very different to that in our study. For example, the kinds of follow-up studies considered in these designs may be subject to consent biases whereby consent is more likely to be given to the follow up of non-coresident parents or children when the level of relationship quality, contact or support, is judged higher by the respondent.

Another strand of literature relates to the reporting of non-coresident children aged under 21 by non-coresident fathers (and sometimes non-coresident mothers), where there is social policy interest in child support payments. This literature does refer to data quality, especially to non-coresident fathers being underreported. One way of assessing reporting of non-coresident children by men is to compare it with reports of non-coresident fathers by custodial mothers as in Sorenson (1997). Differences may be due to underreporting of non-coresident children by men or to differential nonresponse by non-coresident fathers or because non-coresident fathers are more often in institutions not covered by surveys. These kinds of issues may throw some light on sources of errors in our work, although in these studies there is no evidence of errors in individuals reporting from a child perspective and disincentives to provide child support may lead to different kinds of errors for young children compared to adult children. Stykes, Manning and Brown 
(2013) present evidence of "considerable inconsistencies in the estimates of prevalence of non-resident fathers" across US surveys and discuss some potential issues of data quality underlying these inconsistencies.

Also on reporting of non-coresident younger children, Rendall et al. (1999) evaluate the consistency of men's and women's reports of the number of children in retrospective fertility histories collected in BHPS and the US Panel Study of Income Dynamics (PSID). As in our study, the objective is to estimate a common population characteristic, in their case the number of children, from two different sources (mothers and fathers) for subpopulations defined by basic demographic characteristics including marital status and, for PSID, race. They find that while women's reports are consistent with birth registrations, there is a substantial deficit in men's reports of non-coresident children. The authors consider two potential explanations for this discrepancy: underrepresentation of men among survey respondents, due to sampling-frame undercoverage and differential nonresponse, and underreporting by male respondents. Comparisons by subpopulations defined by whether births were nonmarital or from the previous or current marriage reveal in both surveys an underrepresentation of previously married men and underreporting of nonmarital births and births from previous marriages by fathers. Their analysis can be framed as a comparison of reports of the number of parent-child dyads by mothers and fathers but, as in Sorenson (1997) and Stykes, Manning and Brown (2013), reporting of the number of dyads is considered only from the perspective of parents and by subpopulations defined by parent characteristics. We consider the assessment of consistency of reports of the number of parent-child dyads by parents and children for subpopulations defined by both parent and child characteristics.

3. Methodological framework. In this section we set out our methodological framework in generic terms. An illustration of what these terms mean in a concrete application will be given in Section 4 . For example, here we use the generic term 'child' but in the application in Section 4 each child must not be coresident with the parent and must be aged $16+$.

We consider a finite population of $N$ individuals, denoted $U=\{1,2, \ldots, N\}$. Dyads are defined by a dyadic relation $A$ applying to ordered pairs of individuals in $U$. A dyad consists of an ordered pair $(i, j)$ for which the relation holds. We refer to $i$ as the parent and $j$ as the child and write $A_{i j}=1$ if $i$ is a parent of $j$ and $A_{i j}=0$ otherwise.

We suppose that a sample of $n$ respondents, denoted $s$, is drawn from the population $U$. Here, $s$ denotes the set of respondents after sampling and unit non-response have occurred. Each respondent supplies some parent data, 
applying to all dyads to which they belong as a parent, some child data, applying to all dyads to which they belong as a child, and some data about themselves. We suppose that weights can be constructed to enable point estimation of population characteristics using such respondent data.

We suppose that the dyads are not 'nested within sampling', in the sense that if individual $i$ is sampled and belongs to dyad $(i, j)$ or to dyad $(j, i)$ then the other member of the dyad $j$ will not be sampled (or at least this is very unlikely).

We specify population parameters which may be estimated separately from each of the parent and child data and propose to use comparisons of the estimates from these two sources to assess data quality. We begin with the simplest case when, for a given individual $i$, their parent data consists of just their reported value $m_{i}^{(p)}$ of the number of dyads in which they are a parent, that is the number of their children, and the child data consists of just their reported value $m_{i}^{(c)}$ of the number of dyads in which they are a child, that is the number of their parents. We view $m_{i}^{(p)}$ as the reported value of $A_{i+}=\sum_{j \in U} A_{i j}$ and $m_{i}^{(c)}$ as the reported value of $A_{+i}=\sum_{j \in U} A_{j i}$. As a first population parameter which may be estimated from either the parent or child data we consider either $A_{++}=\sum_{i \in U} \sum_{j \in U} A_{i j}$, the total number of dyads in the population, or $\mu . .=A_{++} / N$, the average number of dyads per member of the population. These parameters may be estimated from either the parent or child data since we have

$$
\sum_{i \in U} A_{i+}=\sum_{i \in U} A_{+i}=A_{++}
$$

and we may estimate the population totals in (1) of $A_{i+}$ and $A_{+i}$ by suitable weighted sample totals of $m_{i}^{(p)}$ and $m_{i}^{(c)}$, respectively. For instance, we may estimate $\mu$.. by either

$$
\hat{\mu}_{. .}^{(p)}=\frac{\sum_{i \in s^{(p)}} w_{i}^{(p)} m_{i}^{(p)}}{\sum_{i \in s}(p)} w_{i}^{(p)} \quad \text { or } \quad \hat{\mu}_{. .}^{(c)}=\frac{\sum_{i \in s^{(c)}} w_{i}^{(c)} m_{i}^{(c)}}{\sum_{i \in s^{(c)}} w_{i}^{(c)}}
$$

from the parent data or child data respectively. Here, $s^{(p)}$ and $s^{(c)}$ are defined to be the same as $s$ in the simplest case when both the parent and child data, $m_{i}^{(p)}$ and $m_{i}^{(c)}$, are recorded for all individuals $i$ in the sample $s$. In this case we also set $w_{i}^{(p)}=w_{i}^{(c)}=w_{i}$, a survey weight constructed for the estimation of population totals from totals across $s$. The more general expressions in (2) allow for the weighting to be revised to correct for missing values in $m_{i}^{(p)}$ or $m_{i}^{(c)}$. In this case, $s^{(p)}$ and $s^{(c)}$ denote the subsets of $s$ for which the parent 
and child data, respectively, are observed. In our application, we have no missing values of $m_{i}^{(c)}$ and set $s^{(c)}=s$ and $w_{i}^{(c)}=w_{i}$. We do have missing values of $m_{i}^{(p)}$ and set $s^{(p)} \subset s$ to be the subsample of individuals for whom $m_{i}^{(p)}$ is observed and construct the weight $w_{i}^{(p)}$ to adjust for the missingness in $m_{i}^{(p)}$ as well as sampling.

We propose to assess the consistency of the parent and child data by comparing $\hat{\mu}_{. .}^{(p)}$ and $\hat{\mu}_{. .}^{(c)}$ via the ratio $\hat{\lambda}_{. .}=\hat{\mu}_{. .}^{(p)} / \hat{\mu}_{. .}^{(c)}$. We interpret this ratio as measuring how different, proportionately, the estimated mean number of children is from what would be expected from the estimated mean number of parents. When $\hat{\lambda}_{\text {.. }}=1$ there is no difference. Later, we shall view $\hat{\mu}^{(p)}$ and $\hat{\mu}_{. .}^{(c)}$ as stochastic, subject to sampling and data quality errors, and take $\lambda_{. .}=E\left(\hat{\mu}_{. .}^{(p)}\right) / E\left(\hat{\mu}_{. .}^{(c)}\right)$ as a measure of differential bias in these two estimators. We shall construct confidence intervals for $\lambda$.. and interpret confidence intervals which do not contain 1 as evidence of a data quality problem. This problem could reflect a systematic difference, resulting from measurement error, between $m_{i}^{(p)}$ and $A_{i+}$ or between $m_{i}^{(c)}$ and $A_{+i}$ or both. It could also reflect some kind of systematic non-response or other missingness error, as explained in Section 5.

More generally, we classify the sources of data obtained for individual $i$ as the following:

- Parent data: $m_{i}^{(p)}$ as well as the values of $q$ variables $x_{1 i j}^{(p)}, \ldots, x_{q i j}^{(p)}$ for each child $j=1, \ldots, m_{i}^{(p)}$ of individual $i \in s^{(p)}$;

- Child data: $m_{i}^{(c)}$ as well as the values of $r$ variables $x_{1 i j}^{(c)}, \ldots, x_{r i j}^{(c)}$ for each parent $j=1, \ldots, m_{i}^{(c)}$ of individual $i \in s^{(c)}$;

- Individual data: the values of a series of variables, including all the parent data variables $x_{1}^{(p)}, \ldots, x_{q}^{(p)}$ and all the child data variables $x_{1}^{(c)}, \ldots, x_{r}^{(c)}$, for individual $i \in s$.

To clarify, although the parent data variables $x_{1}^{(p)}, \ldots, x_{q}^{(p)}$ refer to characteristics of children, they have the superscript ${ }^{(p)}$ because they are obtained from the survey respondents' perspectives as parents. Vice versa, although the child data variables $x_{1}^{(c)}, \ldots, x_{r}^{(c)}$ refer to characteristics of parents, they have the superscript $(c)$ because they are obtained from the survey respondents' perspectives as children.

The parent data variables and child data variables may overlap and, indeed, be identical and, in practice, we expect $q$ and $r$ to be small. In our application, both sets of variables consist of sex and age and we have $q=r=2$. In practice, we shall also convert any continuous variable like 
age into a categorical variable, by grouping its values. As a result, we shall convert the parent data variables $x_{1}^{(p)}, \ldots, x_{q}^{(p)}$ into indicator variables for $H$ subpopulations of $U$, denoted $U_{1}^{(p)}, \ldots, U_{H}^{(p)}$, and then, for each $i$, calculate $m_{i h}^{(p)}$, the individual's reported value of $A_{i U_{h}^{(p)}}$, defined as $\sum_{j \in U_{h}^{(p)}} A_{i j}$, for $h=1,2, \ldots, H$. For example, if $U_{h}^{(p)}$ refers to female individuals then $m_{i h}^{(p)}$ will be the individual's reported number of daughters. Similarly, we convert the child data variables $x_{1}^{(c)}, \ldots, x_{r}^{(c)}$ into indicator variables for $K$ subpopulations of $U$, denoted $U_{1}^{(c)}, \ldots, U_{K}^{(c)}$, and then, for each $i$, calculate $m_{i k}^{(c)}$, the individual's reported values of $A_{U_{k}^{(c)} i}$, defined as $\sum_{j \in U_{k}^{(c)}} A_{j i}$, for $k=1,2, \ldots, K$. For example, if $U_{k}^{(c)}$ refers to individuals aged 40-49 then $m_{i k}^{(c)}$ will be the individual's reported number of parents aged 40-49.

We now extend the specification of the population parameter $\mu$.. to alternative choices of subpopulation. The most general case we consider is $\mu_{h k}$, the mean number of dyads per member of the population, where the dyad's child is in subpopulation $U_{h}^{(p)}$ and the dyad's parent is in subpopulation $U_{k}^{(c)}$. We write

$$
\mu_{h k}=\sum_{i \in U_{k}^{(c)}} \sum_{j \in U_{h}^{(p)}} A_{i j} / N .
$$

Two important special cases are: (i) when $U_{k}^{(c)}=U$ and the subpopulation is specified only in terms of $U_{h}^{(p)}$, and we write $\mu_{h} .=\sum_{U_{h}^{(p)}} A_{+j} / N$ and (ii) when $U_{h}^{(p)}=U$ and the subpopulation is specified only in terms of $U_{k}^{(c)}$, and we write $\mu_{\cdot k}=\sum_{U_{k}^{(c)}} A_{i+} / N$.

To estimate the parameter $\mu_{h k}$, or either of its special cases above, from either the parent or child data we make use of the alternative expressions

$$
\mu_{h k}=\sum_{i \in U} I\left(i \in U_{k}^{(c)}\right) A_{i U_{h}^{(p)}} / N=\sum_{i \in U} I\left(i \in U_{h}^{(p)}\right) A_{U_{k}^{(c)} i} / N
$$

for $h=1, \ldots, H, k=1, \ldots, K$. As in (2), we then estimate $\mu_{h k}$ by either

$$
\hat{\mu}_{h k}^{(p)}=\frac{\sum_{i \in s^{(p)}} w_{i}^{(p)} I\left(i \in U_{k}^{(c)}\right) m_{i h}^{(p)}}{\sum_{i \in s^{(p)}} w_{i}^{(p)}} \quad \text { or } \quad \hat{\mu}_{h k}^{(c)}=\frac{\sum_{i \in s^{(c)}} w_{i}^{(c)} I\left(i \in U_{h}^{(p)}\right) m_{i k}^{(c)}}{\sum_{i \in s^{(c)}} w_{i}^{(c)}}
$$

The estimator $\hat{\mu}_{h k}^{(p)}$ is derived from the parent data (to obtain the $m_{i h}^{(p)}$ ), combined with the individual data (to determine which individuals are in 
$\left.U_{k}^{(c)}\right)$, whereas estimator $\hat{\mu}_{h k}^{(c)}$ is derived from the child data (to obtain the $m_{i k}^{(c)}$ ) combined with the individual data (to determine which individuals are in $\left.U_{h}^{(p)}\right)$. For illustration, if $U_{h}^{(p)}$ refers to men and $U_{k}^{(c)}$ refers to women then $\hat{\mu}_{h k}^{(p)}$ is a weighted mean of a variable which is the reported number of sons for women and is 0 for men and $\hat{\mu}_{h k}^{(c)}$ is a weighted mean of a variable which is the reported number of mothers for men and is 0 for women. Expressions for estimators $\hat{\mu}_{h}$. and $\hat{\mu}_{\text {. }}$ of $\mu_{h}$. and $\mu_{\text {.k }}$, respectively, follow naturally from (4) as special cases.

The consistency of the parent and child data may again be assessed by comparing the $\hat{\mu}_{h k}^{(p)}$ and $\hat{\mu}_{h k}^{(c)}$ via the ratio $\hat{\lambda}_{h k}=\hat{\mu}_{h k}^{(p)} / \hat{\mu}_{h k}^{(c)}$ for alternative values of $h$ and $k$. In addition, we may consider the $\hat{\lambda}_{h}=\hat{\mu}_{h}^{(p)} / \hat{\mu}_{h}^{(c)}$ and $\hat{\lambda}_{\cdot k}=$ $\hat{\mu}_{\cdot k}^{(p)} / \hat{\mu}_{\cdot k}^{(c)}$. Differences between these ratios and 1 may indicate measurement error in the reporting of $A_{i U_{h}^{(p)}}$ by $m_{i h}^{(p)}$ or in the reporting of $A_{U_{k}^{(c)} i}$ by $m_{i k}^{(c)}$.

In order to estimate standard errors for the ratios $\hat{\lambda}_{h k}, \hat{\lambda}_{h}, \hat{\lambda}_{\cdot k}$ and $\hat{\lambda}_{\text {.., }}$, as well as their component mean estimators, such as $\hat{\mu}_{h k}^{(p)}$ and $\hat{\mu}_{h k}^{(c)}$, we shall use linearization variance estimation. We shall take account of the nature of the weights $w_{i}^{(p)}$ and $w_{i}^{(c)}$ as well as the nature of the sampling scheme and sources of missing data according to which the samples $s^{(p)}$ and $s^{(c)}$ are determined from $U$. Further detail for our application is given in Section 4.3 and the supplementary materials. A 95\% confidence interval for a parameter $\lambda$ corresponding to an estimator $\hat{\lambda}$ and standard error s.e. $(\hat{\lambda})$ will be constructed using standard normal theory as $\hat{\lambda} \pm 1.96$ s.e. $(\hat{\lambda})$.

\section{Data and application.}

4.1. Overview of the British Household Panel Survey and data from the family networks module. The British Household Panel Survey (BHPS) began in 1991 with a sample of around 5,500 households and 10,300 individuals from Great Britain (ISER, 2018). These have been followed up since with annual waves of data collection. At wave 18 BHPS participants were asked to join a larger survey Understanding Society, also known as the UK Longitudinal Household Study (UKHLS), and first interviews with those BHPS participants joining the new survey took place in 2010-11. Information on relatives living outside a respondent's household was collected as part of the family networks module. The questionnaire has been subject to some rotation, with the module being administered in 2001, 2006 (BHPS waves 11 and 16) and bienially thereafter in 2011-13, 2013-15 and 2015-17 (UKHLS waves 3,5 and 7 ). In this paper, we analyse data from the 2001 module which allows the use of 2001 census data for poststratification adjustment 
(see Section 6). Analysis of the first wave in the series may also serve as a baseline for future longitudinal analyses.

We shall consider dyads as parent/child non-coresident pairs among the population of all individuals aged $16+$ living in GB. The age restriction is consistent with BHPS eligibility criteria for adult sample members, and hence only dyads with an adult child are considered. Although the initial BHPS sample consists of individuals in private households, original sample members (OSMs) who moved into institutional accommodation (including student halls of residence and nursing homes but not prisons) were followed where possible.

Data about non-coresident children and parents are obtained from questions about the presence of children and parents living outside the household. As in Section 3, we refer to information collected from respondents about their parents, that is from the perspective of the child, as 'child data'. Similarly, information collected about children (from parent respondents) is referred to as 'parent data'. The child data include indicators of whether the respondent has a non-coresident mother (defined in the question as not "living in this household with you at the moment"), whether they have a non-coresident father, and the ages of such parents. In BHPS, no distinction is made between biological, step or adoptive parents and thus, in the notation of Section $3, m_{i}^{(c)}$ can take only three values: 0,1 or 2 . We have $r=2$ child variables, sex and age. The parent data are restricted to a question about the number of non-coresident children. As for the child data, no distinction is made between biological, step and adopted children. An important limitation of the parent data is that the age and sex composition of non-coresident children was not collected. The lack of information on children's age is especially problematic because the focus on adult dyads implies that only adult children should be included in the count of non-coresident children. Moreover, age and sex of children is required for disaggregated analyses by demographic characteristics of parents and children. For these reasons, we use derived measures of the number of non-coresident children, by age and sex, rather than direct reports of the total number.

4.2. Derivation of the number of non-coresident children and weighting for incomplete birth histories. The number of non-coresident children is calculated as the difference between the total number of children born by wave 11 and the number of biological children coresident at wave 11 . The total number born is derived from a combination of retrospective child histories collected at wave 2 and panel data from the household enumeration for waves 3 to 11, while the number coresident at wave 11 comes from the 
household enumeration at that wave.

Retrospective birth histories were collected for almost all sample members interviewed at wave 2 . There was no further attempt to collect histories from the main BHPS sample after wave 2. In particular, histories were not collected from permanent and temporary sample members (PSMs and TSMs) who entered the panel after wave 2 or from children of OSMs who became OSMs themselves when they reached age 16. Hence the derived measure of the number of non-coresident children is left-truncated for most PSMs and TSMs and for the youngest OSMs. Histories were collected at waves 11 and 12 for Welsh, Scottish and Northern Ireland extension sample members (who joined at waves 9 and 11), but they were not asked the family network questions. Members of the European Community Household Panel (ECHP) survey low-income sub-sample (who joined at wave 7) were also unlikely to be asked for a birth history. We therefore restrict our analysis sample $s^{(p)}$ for the parent data to BHPS OSMs and weight to adjust for the higher rate of missing data among young OSMs. As the probability of having a missing history is unrelated to the reported number of non-coresident children after controlling for age, six age-based weighting classes were used: $<30,30-39$, 40-49, 50-59, 60-69 and 70+ years, denoted $a=1, \ldots, 6$. The weight $w_{i}^{(p)}$ in Section 3 is taken as $n_{a} / n_{a}^{(p)}$ if $i$ is in age group $a$, where $n_{a}$ and $n_{a}^{(p)}$ are the numbers of respondents in $s^{(p)}$ and $s$ respectively. These samples are discussed further in Section 4.3. The proportion of cases with complete data ranges from 0.33 for age $<30$ to $0.94-0.98$ for the older age groups. The unweighted mean of the reported number of non-coresident children for the full sample of BHPS OSMs shows close agreement to the weighted mean for the sub-sample with complete histories (0.950 vs 0.949).

A comparison of the derived measure of the total number of non-coresident children with the reported measure (among those with a complete birth history) shows an exact match in $87 \%$ of cases, and a discrepancy of 1 child for a further $10 \%$. We also considered a derived measure that includes step and adopted children because these children may be included in parental reports of non-coresident children; however, their inclusion led to a slight reduction in the proportion with an exact match to $85 \%$. For this reason, and because of a large amount of missing data on the dates of birth for these children, the number of biological children is used in all analysis.

4.3. Analysis sample. The family network questions were asked of 9724 of the wave 11 respondents. The module was not administered among the Welsh, Scottish and Northern Ireland extension samples, nor proxy and telephone interviewees. All but two respondents answered the questions about 
TABLE 1

Frequency distributions of numbers of non-coresident children $\left(m_{i}^{(p)}\right)$ and non-coresident parents $\left(m_{i}^{(c)}\right)$

\begin{tabular}{|lrlr|}
\hline$m_{i}^{(p)}$ & Frequency & $m_{i}^{(c)}$ & Frequency \\
\hline 0 & 2998 & & 3161 \\
1 & 833 & 1 & 1530 \\
2 & 1053 & & 2177 \\
3 & 535 & & Total \\
\cline { 3 - 4 } 4 & 164 & & 6868 \\
$5+$ & 105 & & \\
\hline Total & 5688 & & \\
\hline
\end{tabular}

the presence of non-coresident parents and children. Of these, we restrict the analysis sample to the 6961 BHPS OSMs. The following sample members were excluded because of high probabilities of incomplete birth histories: 623 PSMs, 741 TSMs, and 1397 ECHP members. The size of the analysis sample, denoted $s$ in Section 3, reduces to 6868 after the following further exclusions: 11 respondents aged 15, 74 who provided a birth history but with missing data on the date of birth or sex of at least one child, and 8 with missing data for parental age. Of this sample, 5688 provided a birth history at wave 2. In the notation of Section 3, this subsample is denoted $s^{(p)}$, whereas the subsample $s^{(c)}$ is identical to $s$. Table 1 shows the frequency distributions of the number of non-coresident children aged 16+ (among those who provided a birth history) and the number of non-coresident parents for the analysis sample.

The sample $s$ is based on a stratified multi-stage sample of OSMs in 1991 (wave 1). The primary sampling units (PSUs), denoted $c$, consist of 250 postcode sectors. As an approximation to the stratification of PSUs, we take the strata, denoted $\ell$, as the $L=18$ regions employed as major strata. Sample inclusion probabilities of OSMs in 1991 were virtually identical. Weights were constructed to handle nonresponse at wave 1 , and successive attrition at waves 2 to 11 (Taylor et al., 2010). We comment further on these in Section 6 and the supplementary materials. For variance estimation, we employ linearization, approximating point estimators by sample sums $\sum_{s} z_{i}$ of appropriate linearized variates $z_{i}$, derived in the supplementary materials for the various estimators considered in Section 3. We then use a standard ultimate cluster linearization variance estimator for the stratified multistage sampling design (Valliant, Dever and Kreuter, 2013, p.404), given by

$$
v=\sum_{\ell=1}^{L} \frac{n_{\ell}}{n_{\ell}-1} \sum_{c=1}^{n_{\ell}}\left(z_{\ell c}-\bar{z}_{\ell}\right)^{2},
$$


where $n_{\ell}$ is the number of PSUs in stratum $\ell, z_{\ell c}=\sum_{i \in s_{\ell c}} z_{i}, \overline{z_{\ell}}=n_{\ell}^{-1} \sum_{c=1}^{n_{\ell}} z_{\ell c}$ and $s_{\ell c}$ denotes the set of individuals $i \in s$ falling into PSU $c$ in stratum $\ell$. We allow not only for clustering and stratification but also for variation arising from sample dependence of the weights. Importantly, we allow for correlation between estimates based on the parent and child data sources, such as $\hat{\mu}_{h k}^{(p)}$ and $\hat{\mu}_{h k}^{(c)}$. Missingness of a birth history, as described in Section 4.2 , is represented by an indicator denoted $u_{i}$ so that, for example, $s^{(p)}=\left\{i \in s \mid u_{i}=1\right\}$. For variance estimation, $u_{i}$ is treated as fixed so no stochastic assumption is made about the nature of this source of missingness. We do assume independence of individual-level missiness and reporting error between PSUs.

5. Comparison of estimates from parent and child data. We now present some initial comparisons of the estimates $\hat{\mu}^{(p)}$ and $\hat{\mu}^{(c)}$ of the mean number of dyads per member of the population, both overall and for those dyads where the parent and/or child belong to different subpopulations defined by sex or age group. In this section, the $\hat{\mu}^{(p)}$ are only weighted to adjust for the higher rate of incomplete birth histories for young respondents, while the $\hat{\mu}^{(c)}$ are unweighted. Hence, in $(2), w_{i}^{(p)}$ consists of the simple weight described in Section 4.2 and $w_{i}^{(c)}$ is taken as a constant. Table 2 shows the two estimates of the overall parameter $\mu_{\text {.. }}$, defined below (1), and subpopulation parameters $\mu_{h}, \mu_{\cdot k}$ and $\mu_{h k}$, defined in and below (3), for subpopulations defined by sex. Their ratios (denoted $\hat{\lambda}_{. .}, \hat{\lambda}_{h}, \hat{\lambda}_{. k}$ or $\hat{\lambda}_{h k}$ in Section 3) appear in the final column. Standard errors are given in parentheses.

The ratio of the estimates of the overall parameter $\mu$.. (1.019) differs from 1 by less than a standard error (0.033) and the $95 \%$ confidence interval for $\lambda$. is $(0.95,1.08)$. This finding therefore provides no evidence that the number $m_{i}^{(p)}$ of non-coresident children aged $16+$ reported in the parent data differs systematically from what would be expected given the number $m_{i}^{(c)}$ of non-coresident parents reported in the child data.

The corresponding findings for the different subpopulations in Table 2 mostly lead to a different conclusion, however. Only one of the $95 \%$ confidence intervals for the ratio $\lambda$ for subpopulations defined by sex, $(0.88,1.02)$ for fathers, $(1.01,1.15)$ for mothers, $(1.07,1.24)$ for sons and $(0.85,0.98)$ for daughters, contains 1 . Among the $95 \%$ confidence intervals for the parameters $\lambda_{h k}$ for the four subpopulations defined by sex of both parents and children only two contain 1 .

A striking feature of the results for subpopulations in Table 2 is that the ratios $\hat{\lambda}_{h}$. and $\hat{\lambda}_{\cdot k}$ differ from 1 in opposite directions, both for fathers vs. mothers ( $k=1$ vs. 2 ) and for sons vs. daughters ( $h=1$ vs. 2$)$. These 
TABLE 2

Estimates of means of numbers of non-coresident children aged $16+$ and parents and their ratio: overall and by subpopulation. Standard errors in parentheses. Ratios for which the $95 \%$ confidence interval does not contain 1 are in bold.

\begin{tabular}{|lccccccc|}
\hline Subpopulation (parameter) & \multicolumn{2}{c}{$\hat{\mu}^{(p)}$} & \multicolumn{3}{c}{$\hat{\mu}^{(c)}$} & \multicolumn{2}{c}{$\hat{\lambda}=\hat{\mu}^{(p)} / \hat{\mu}^{(c)}$} \\
\hline Overall $(\mu .)$. & 0.873 & $(0.019)$ & 0.857 & $(0.013)$ & 1.019 & $(0.033)$ \\
& & & & & & \\
Sex of parents & & & & & & \\
$\quad$ Fathers $(\mu \cdot 1)$ & 0.362 & $(0.010)$ & 0.382 & $(0.007)$ & 0.948 & $(0.037)$ \\
$\quad$ Mothers $(\mu \cdot 2)$ & 0.511 & $(0.013)$ & 0.475 & $(0.007)$ & $\mathbf{1 . 0 7 6}$ & $(0.035)$ \\
& & & & & & \\
Sex of children & & & & & & \\
$\quad$ Sons $\left(\mu_{1}.\right)$ & 0.439 & $(0.012)$ & 0.381 & $(0.009)$ & $\mathbf{1 . 1 5 2}$ & $(0.043)$ \\
Daughters $\left(\mu_{2 .}\right)$ & 0.434 & $(0.012)$ & 0.475 & $(0.009)$ & $\mathbf{0 . 9 1 2}$ & $(0.033)$ \\
& & & & & & \\
Sex of both parents and children & & & & & & \\
$\quad$ Fathers/sons $\left(\mu_{11}\right)$ & 0.182 & $(0.006)$ & 0.172 & $(0.005)$ & 1.054 & $(0.048)$ \\
Fathers/daughters $\left(\mu_{21}\right)$ & 0.181 & $(0.006)$ & 0.210 & $(0.005)$ & $\mathbf{0 . 8 6 1}$ & $(0.039)$ \\
$\quad$ Mothers/sons $\left(\mu_{12}\right)$ & 0.258 & $(0.008)$ & 0.209 & $(0.005)$ & $\mathbf{1 . 2 3 3}$ & $(0.047)$ \\
Mothers/daughters $\left(\mu_{22}\right)$ & 0.253 & $(0.008)$ & 0.266 & $(0.005)$ & 0.952 & $(0.036)$ \\
\hline
\end{tabular}

differences are quite strong and greater (in absolute size) than the difference between $\hat{\lambda}$.. and 1 for the overall population. There seem to us to be two main possible reasons for these differences, either reporting error or missing data. We contend that the latter is much more plausible than the former.

Consider first reporting error and the 'sex of children' results. Based on the non-coresident father literature mentioned in Section 2, the most plausible form of reporting error would seem to be underreporting (rather than overreporting). Suppose that non-coresident children, i.e. sons and daughters, and their total $m_{i}^{(p)}$, are underreported, but that non-coresident parents are correctly reported then we would expect $\hat{\lambda}_{h}$. to be less than 1 for both sons $(h=1)$ and daughters $(h=2)$, even if the degree of underreporting (and hence $\lambda_{h}$.) differs between sons and daughters. This is not consistent with our observation that the ratio is above 1 for sons. Similarly, if non-coresident parents are underreported but non-coresident children are correctly reported then we would expect $\hat{\lambda}_{h}$. to be greater than 1 for both sons and daughters, even if the degree of underreporting (and hence $\hat{\lambda}_{h}$.) differs between sons and daughters. Again, this is not consistent with our observation that the ratio is below 1 for daughters. The only way of obtaining the opposite direction of departures of $\hat{\lambda}_{h}$. from 1 for sons and daughters would seem to be if some fairly extreme interaction occurred, such as parents underreporting daughters but not sons and male but not female respondents underreporting parents. This seems to us fairly implausible. 
On the other hand, consider the possibility of nonresponse or a similar kind of missing data. As a simple model to explain the patterns of results, suppose that male and female individuals are missing from the sample with probabilities $1-q_{m}$ and $1-q_{f}$, respectively. In the case of nonresponse, $q_{m}$ and $q_{f}$ may be interpreted as response propensities. Let $A_{m m}, A_{f m}$, $A_{m f}$ and $A_{f f}$ denote the numbers of father-son, mother-son, father-daughter and mother-daughter dyads, respectively, and let $A_{+m}=A_{m m}+A_{f m}$ and $A_{+f}=A_{m f}+A_{f f}$. Also let $N_{m}$ and $N_{f}$ denote the numbers of male and female individuals, respectively, in the population. Assuming that reporting errors are absent, we obtain in the supplementary materials the following expressions for the approximate expectations of the relevant estimators.

When the subpopulation $U_{h}^{(p)}$ refers to sons:

$$
E\left[\hat{\mu}_{h .}^{(p)}\right] \approx \frac{q_{m} A_{m m}+q_{f} A_{f m}}{q_{m} N_{m}+q_{f} N_{f}} \quad \text { and } \quad E\left[\hat{\mu}_{h .}^{(c)}\right] \approx \frac{q_{m} A_{+m}}{q_{m} N_{m}+q_{f} N_{f}}
$$

so that

$$
E\left[\hat{\mu}_{h .}^{(p)}-\hat{\mu}_{h .}^{(c)}\right] \approx \frac{-\left(q_{m}-q_{f}\right) A_{f m}}{q_{m} N_{m}+q_{f} N_{f}}
$$

When the subpopulation $U_{h}^{(p)}$ refers to daughters:

$$
E\left[\hat{\mu}_{h .}^{(p)}\right] \approx \frac{q_{m} A_{m f}+q_{f} A_{f f}}{q_{m} N_{m}+q_{f} N_{f}} \quad \text { and } \quad E\left[\hat{\mu}_{h \cdot}^{(c)}\right] \approx \frac{q_{f} A_{+f}}{q_{m} N_{m}+q_{f} N_{f}}
$$

so that

$$
E\left[\hat{\mu}_{h .}^{(p)}-\hat{\mu}_{h .}^{(c)}\right] \approx \frac{\left(q_{m}-q_{f}\right) A_{m f}}{q_{m} N_{m}+q_{f} N_{f}} .
$$

The estimators $\hat{\mu}_{h}^{(p)}$ and $\hat{\mu}_{h}^{(c)}$. considered here are in the form of ratios $\hat{\phi} / \hat{\psi}$ and we assume an asymptotic framework in which such ratios minus the ratio of their expectations $E(\hat{\phi}) / E(\hat{\psi})$ converge to zero in probability. The approximation notation $\approx$ above is used to denote this limiting behaviour so that differences between the left and right hand sides of $\approx$ converge to zero in probability. Expectations and this limiting behavior are with respect to sampling (individuals are assumed to be sampled with equal probability), the simple model for missingness above and, in the case of $\hat{\mu}_{h \text {. }}^{(p)}$, the process which generates missing child histories (individuals in the same age group are assumed to have equal probabilities of missingness).

If missingness is unrelated to sex and $q_{m}=q_{f}$ then the expressions in both (6) and (7) are zero and we would expect the ratio $\hat{\lambda}_{h}$. to be 1 , subject 
to sampling error. In the more realistic case, however, when $q_{m}$ and $q_{f}$ are unequal, the differences in (6) and (7) are of opposite signs (since both $A_{f m}$ and $A_{m f}$ are positive) and we expect the ratios $\hat{\lambda}_{h}$. for sons and daughters to be opposite sides of 1 , as we observe. The finding in Table 2 that the difference $\hat{\mu}_{h}^{(p)}-\hat{\mu}_{h}^{(c)}$ is positive for sons corresponds in expectation to $q_{m}-q_{f}$ being negative in (6), that is to the response rate being lower for men than women. Indeed, a comparison of sample and census counts by sex gives an estimate of $q_{m} / q_{f}$ of 0.90 .

Moreover, we can compare the magnitudes of (6) and (7) by noting that $A_{f m}-A_{m f}=\left(\pi_{\cdot m}-\pi_{m}\right) A_{++}$under the approximation that the sexes of children and parents are independent (so that e.g. $A_{m f}=A_{m+} A_{+f} / A_{++}$), where $\pi_{m} \cdot=A_{m+} / A_{++}$and $\pi_{\cdot m}=A_{+m} / A_{++}$are the proportions of dyads where the parent or child, respectively, is male. Since we expect $\pi_{m}$. $<\pi_{\cdot m}$ when the proportion male gradually declines with age, as we observe it does using the census data, it follows that we expect $A_{f m}>A_{m f}$ and so we expect the absolute difference between $\hat{\lambda}_{h}$. and 1 to be greater for sons than daughters, as we observe in Table 2 ( $15 \%$ vs $9 \%$ ).

Corresponding results are obtained in the supplementary materials for subpopulations defined by fathers and mothers. Under the assumption, as before, that the response rate is lower for men than women $\left(q_{m}<q_{f}\right)$, we show that, in expectation, $\hat{\mu}_{. k}^{(p)}$ is less than $\hat{\mu}_{. k}^{(c)}$ and hence the ratio $\hat{\lambda}_{\cdot k}$ is expected to be less than 1 for fathers, as indeed we observe. Similarly, we show that the ratio is expected to be greater than 1 for mothers, as observed. The positions of the sexes are reversed in this theory, with the ratio for fathers expected to be lower than for mothers in contrast to the sons having a higher expected ratio than daughters and this reversal is what is observed empirically in Table 2. We also find, under the assumptions that sexes of children and parents are independent and $\pi_{m} \cdot<\pi_{\cdot m}$, that the absolute difference between $\hat{\lambda}_{\cdot k}$ and 1 is expected to be greater for mothers than fathers, as we observe in Table 2 ( $8 \%$ vs $5 \%$ ).

We may also study the overall estimators $\hat{\mu}_{. .}^{(p)}$ and $\hat{\mu}_{. .}^{(c)}$ under this simple theoretical model for nonresponse. We find that

$$
E\left[\hat{\mu}_{. \cdot}^{(p)}\right]-E\left[\hat{\mu}_{. \cdot}^{(c)}\right] \approx \frac{\left(q_{m}-q_{f}\right)\left(\pi_{m} \cdot-\pi_{\cdot m}\right) A_{++}}{q_{m} N_{m}+q_{f} N_{f}} .
$$

Under our assumptions above that $q_{m}<q_{f}$ and $\pi_{m}$. $<\pi_{\cdot m}$, this difference must be positive and we expect the ratio $\hat{\lambda}_{. .}=\hat{\mu}_{. .}^{(p)} / \hat{\mu}_{.}^{(c)}$ to be greater than 1 , as indeed we observe in Table 2.

We now present results for subpopulations defined by age of parents and age of children (Table 3). As for the analysis by sex of parents and children, 
TABLE 3

Estimates of means of numbers of non-coresident children aged $16+$ and parents and their ratio by age of parents and children. Standard errors in parentheses. Ratios for which the $95 \%$ confidence interval does not contain 1 are in bold.

\begin{tabular}{|lcccccc|}
\hline Subpopulation & \multicolumn{2}{c}{$\hat{\mu}^{(p)}$} & \multicolumn{2}{c}{$\hat{\mu}^{(c)}$} & $\hat{\lambda}=\hat{\mu}^{(p)} / \hat{\mu}^{(c)}$ \\
\hline Age of parent & & & & & & \\
$<50$ & 0.089 & $(0.005)$ & 0.080 & $(0.005)$ & 1.117 & $(0.063)$ \\
$50-59$ & 0.263 & $(0.011)$ & 0.219 & $(0.009)$ & $\mathbf{1 . 2 0 2}$ & $(0.057)$ \\
$60-69$ & 0.233 & $(0.011)$ & 0.253 & $(0.008)$ & 0.924 & $(0.051)$ \\
$70-79$ & 0.193 & $(0.011)$ & 0.208 & $(0.007)$ & 0.928 & $(0.066)$ \\
$80+$ & 0.094 & $(0.007)$ & 0.097 & $(0.005)$ & 0.967 & $(0.090)$ \\
& & & & & & \\
Age of child & & & & & & \\
$16-19$ & 0.035 & $(0.003)$ & 0.032 & $(0.003)$ & 1.066 & $(0.089)$ \\
$20-24$ & 0.084 & $(0.005)$ & 0.078 & $(0.005)$ & 1.073 & $(0.052)$ \\
$25-29$ & 0.127 & $(0.006)$ & 0.106 & $(0.006)$ & $\mathbf{1 . 1 9 6}$ & $(0.058)$ \\
$30-39$ & 0.312 & $(0.012)$ & 0.290 & $(0.011)$ & 1.073 & $(0.058)$ \\
$40-49$ & 0.202 & $(0.010)$ & 0.232 & $(0.009)$ & $\mathbf{0 . 8 7 2}$ & $(0.057)$ \\
$50+$ & 0.124 & $(0.008)$ & 0.118 & $(0.005)$ & 1.051 & $(0.090)$ \\
\hline
\end{tabular}

the ratios deviate from 1 to a much greater degree than the $2 \%$ deviation overall. We observe that for both age of parents and children, the ratios follow a similar pattern to that observed for sex, with some ratios differing significantly from 1 both above and below. For age of parent groups, the ratio is above 1 for ages up to 59 and below 1 from age 60; for age of child groups, the ratio is above 1 up to age 39 and below 1 for $40-49$. (The ratio is above 1 again for children aged $50+$, but the deviation is within 1 standard error for this small group.) As age of parents and children is positively correlated, the ratios are not expected to differ from 1 in opposite directions for parent age and child age.

To summarize this initial analysis, although we observe a value 1.02 (se $=0.03)$ for the overall ratio $\hat{\lambda}_{. .}=\hat{\mu}_{. .}^{(p)} / \hat{\mu}_{. .}^{(c)}$ that is not far from 1 , we observe more substantial deviations from 1 in different subpopulations defined by sex and age. We do not find that the patterns of these deviations correspond in a very plausible way with any obvious simple model for reporting error. On the other hand, they do seem to correspond remarkably well with a simple model for differential nonresponse. Hence, our priority in the next section will be to consider nonresponse further and the correction of potential nonresponse bias via weighting. We return to considering reporting error in Section 7.

6. Weighting adjustments. The analysis in Section 5 suggested that a simple partial explanation for the deviations of the observed ratios from 
1 was that population units were differentially represented in the analysis sample with respect to sex and age. In this section we consider an alternative choice of weights $w_{i}^{(p)}$ and $w_{i}^{(c)}$ in (2) and (4) to adjust for such differential missingness.

We considered four options, derived from weights supplied with the BHPS data (Taylor et al., 2010), designed to compensate for nonresponse, and weights we constructed using estimates from the 2001 census in GB, designed to compensate for differential representation of the 2001 population in the analysis sample. The options are described in the supplementary material together with a theoretical and empirical comparison. Taking our primary objective as the reduction of the discrepancies between the ratios and 1, we concluded that the most suitable of our options was to employ poststratification based on population counts in 24 age-sex groups (poststrata) from the census. The age groups were 16-29, 5-year groups for ages $30-79$, and $80+$. Denoting the poststrata by $b=1, \ldots, 24$, the poststratified weights are

$$
w_{P S i}^{(p)}=\frac{n}{N} \frac{N_{b}}{n_{b}^{(p)}}, \quad w_{P S i}^{(c)}=\frac{n}{N} \frac{N_{b}}{n_{b}} \quad \text { if } i \text { is in poststratum } b,
$$

where $n_{b}$ and $n_{b}^{(p)}$ are the numbers of respondents in poststratum $b$ in samples $s$ and $s^{(p)}$, respectively, with $n=\sum n_{b}$, and $N_{b}$ is the census count in poststratum $b$, with $N=\sum N_{b}$. Values of the poststratified weights are given in the supplementary materials. Since the poststrata are nested within the six age-based weighting classes used in Section 4.2 to construct the weighting class adjustment applied in Section 5, the poststratified weights incorporate this adjustment implicitly.

Table 4 contains estimates $\hat{\mu}^{(p)}, \hat{\mu}^{(c)}$ and $\hat{\lambda}$ with standard errors obtained using poststratified weights. We observe first that the standard errors are generally much reduced compared with the standard errors in Tables 2 and 3. Age and sex of respondents clearly play a key role in the parameters of interest and respondents' age will be highly correlated with the ages of their parents and children so such a gain in efficiency from age-sex poststratification seems plausible. Ratios $\hat{\lambda}$ for which their associated $95 \%$ confidence intervals do not contain 1 are highlighted in bold. We treat such cases as evidence of bias in the sense that $\hat{\mu}^{(p)}$ and $\hat{\mu}^{(c)}$ are estimating different quantities. We focus in the remainder of this section on such cases and the largest deviations of $\hat{\lambda}$ from 1 .

We consider subpopulations defined by sex and age in turn, beginning with ones defined by sex of parents and/or children. We observe that the values of $\hat{\lambda}$ in Table 4 for these subpopulations are all closer to 1 than their initial estimates in Table 2 . The poststratification adjustment has moved the 
TABLE 4

Poststratified estimates of means of numbers of non-coresident children aged 16+ and parents and their ratio by subpopulation. Standard errors in parentheses. Ratios for which the $95 \%$ confidence interval does not contain 1 are in bold.

\begin{tabular}{|c|c|c|c|c|c|c|}
\hline \multirow{2}{*}{$\begin{array}{l}\text { Subpopulation } \\
\text { Overall }\end{array}$} & \multicolumn{2}{|c|}{$\hat{\mu}^{(p)}$} & \multicolumn{2}{|c|}{$\hat{\mu}^{(c)}$} & \multicolumn{2}{|c|}{$\hat{\lambda}=\hat{\mu}^{(p)} / \hat{\mu}^{(c)}$} \\
\hline & 0.854 & $(0.014)$ & 0.861 & $(0.009)$ & 0.992 & $(0.019)$ \\
\hline \multicolumn{7}{|l|}{ Sex of parents } \\
\hline Fathers & 0.372 & $(0.007)$ & 0.388 & $(0.005)$ & 0.958 & $(0.022)$ \\
\hline Mothers & 0.483 & $(0.010)$ & 0.474 & $(0.005)$ & 1.019 & $(0.022)$ \\
\hline \multicolumn{7}{|l|}{ Sex of children } \\
\hline Sons & 0.430 & $(0.010)$ & 0.415 & $(0.006)$ & 1.036 & $(0.026)$ \\
\hline Daughters & 0.424 & $(0.010)$ & 0.446 & $(0.006)$ & 0.950 & $(0.025)$ \\
\hline \multicolumn{7}{|c|}{ Sex of both parents and children } \\
\hline Fathers/sons & 0.186 & $(0.005)$ & 0.189 & $(0.004)$ & 0.986 & $(0.030)$ \\
\hline Fathers/daughters & 0.186 & $(0.006)$ & 0.199 & $(0.003)$ & 0.932 & $(0.031)$ \\
\hline Mothers/sons & 0.244 & $(0.006)$ & 0.227 & $(0.004)$ & 1.077 & $(0.030)$ \\
\hline Mothers/daughters & 0.239 & $(0.006)$ & 0.247 & $(0.003)$ & 0.965 & $(0.028)$ \\
\hline \multicolumn{7}{|l|}{ Age of parents } \\
\hline$<50$ & 0.082 & $(0.004)$ & 0.084 & $(0.004)$ & 0.984 & $(0.058)$ \\
\hline $50-59$ & 0.236 & $(0.007)$ & 0.238 & $(0.007)$ & 0.990 & $(0.035)$ \\
\hline $60-69$ & 0.243 & $(0.007)$ & 0.253 & $(0.006)$ & 0.961 & $(0.034)$ \\
\hline $70-79$ & 0.190 & $(0.007)$ & 0.195 & $(0.006)$ & 0.976 & $(0.045)$ \\
\hline $80+$ & 0.103 & $(0.005)$ & 0.092 & $(0.004)$ & 1.120 & $(0.068)$ \\
\hline \multicolumn{7}{|l|}{ Age of children } \\
\hline $16-19$ & 0.033 & $(0.003)$ & 0.034 & $(0.002)$ & 0.972 & $(0.080)$ \\
\hline $20-24$ & 0.079 & $(0.004)$ & 0.082 & $(0.003)$ & 0.968 & $(0.052)$ \\
\hline $25-29$ & 0.118 & $(0.005)$ & 0.111 & $(0.003)$ & 1.068 & $(0.047)$ \\
\hline 30-39 & 0.300 & $(0.008)$ & 0.316 & $(0.004)$ & 0.950 & $(0.028)$ \\
\hline $40-49$ & 0.204 & $(0.007)$ & 0.209 & $(0.004)$ & 0.979 & $(0.038)$ \\
\hline $50+$ & 0.130 & $(0.006)$ & 0.110 & $(0.003)$ & 1.179 & $(0.067)$ \\
\hline
\end{tabular}


estimates close enough to 1 for the confidence intervals for the main effects (father, mother, son, daughter) to contain 1, but not quite close enough for this to hold for the mothers/sons or fathers/daughters effects (even though their point estimates are greatly improved).

In summary, the poststratification adjustment has been very effective in removing differential bias for the subpopulations defined by sex, but there is still evidence that it has not been wholly successful. This suggests either an element of misspecification of the underlying missingness model or an effect of reporting error. We address the second possibility in the next section. Here we consider the possibility of departures from the implicit model underlying the poststratification, that missingness depends at most on the sex and age of the member of the dyad included in the sample. In particular, it is assumed that, given these characteristics, missingness does not depend on the sex of the other non-sampled member of the dyad.

To accommodate departures from these missingness assumptions, we might consider a poststratification or calibration adjustment which controls for other population characteristics in addition to sex and age. Characteristics of the dyadic population are not available to us. A variable such as household size would be available from the 2001 census but its use for adjustment would depend on it being measured the same in the census and the survey data and we shall not pursue this possibility any further here. Another approach follows from noting that the poststratification adjusts not only for non-response but also for coverage error, since the 2001 census data includes individuals who were not resident in GB in 1991, such as those who migrated to GB after that year and had zero chance of inclusion in the analysis sample. Hence another possible explanation for the discrepant results for mother-son dyads and father-daughter dyads might be a distorting effect of the coverage element of the weighting adjustment. To explore the possibility of such an effect we might study the impact of modifying the population used in the poststratification weight. We shall do this below for age but we have not identified any rationale for such a modification for these two sex-based subpopulations.

Consider then subpopulations defined by age of parents or children. We observe in Table 4 that the greatest deviations of the ratio from 1 occur in the eldest groups, parents of $80+$ or children of $50+$. To help understand these deviations we have also undertaken poststratification using 2001 census counts for just the household population, that is excluding individuals living outside private households, such as in care homes. Such individuals were included when calculating the poststratified weights employed in Table 4. We find that poststratification by the household population leads to es- 
timates $\hat{\mu}^{(p)}$ (0.092 and 0.120 for parents aged $80+$ and children aged 50+, respectively) closer to the initial estimates (0.094 and 0.124$)$ than to poststratification by the total population $(0.103$ and 0.130$)$. We attribute this to the larger weights $w_{i}^{(p)}$ for the $80+$ age groups when the total (rather than the household) population is used, because of the importance of individuals aged $80+$ living outside the household population, especially in care homes. On the other hand, we find that poststratification by the household population leads to estimates $\hat{\mu}^{(c)}(0.093$ and 0.112 for parents aged $80+$ and children aged $50+$, respectively) closer to poststratification by the total population (0.092 and 0.110 ) than to the initial estimates (0.097 and 0.118). We attribute this to the fact that there is little difference between these two poststratification weights $w_{i}^{(c)}$ for those age groups (ages $50-69$, say) who are most likely to have elderly parents. Combining the effects on $\hat{\mu}^{(p)}$ and $\hat{\mu}^{(c)}$, we find that poststratification by the household population leads to ratios $\hat{\lambda}$ that are closer to 1 (0.984 and 1.074 for parents aged $80+$ and children aged $50+$ respectively) and with confidence intervals that contain 1 in both cases. In short, we consider that the deviations of $\hat{\lambda}$ from 1 for the eldest groups in Table 4 can be attributed particularly to underrepresentation of the elderly in care homes in the BHPS.

The poststratified weights measure the extent to which the analysis sample is underrepresented in the different age-sex groups. Of the four largest poststratified weights, one is for the oldest age group (women aged $80+$ ), as could be anticipated from the discussion above, but the remaining three are for younger age groups (men aged 30-34, 35-39 and women aged 25-29). This may reflect net immigration to GB between 1991 and 2001. Hatton (2005) showed higher net immigation over the 1990s for the 15-24 and 25-44 age groups (and age in 2001 will be higher than age at immigration). Rendall and Ball (2004) found that a large proportion of the overseas-born population in the 2001 census were in their 20s, 30s and 40s. Undercoverage of immigrants in the analysis sample may therefore be expected to lead to increases in $\hat{\mu}_{h}^{(c)}$. for poststratified (vs. initial) weights for such age groups but have rather less impact on corresponding values of $\hat{\mu}_{h}^{(p)}$. Indeed, we find that the two largest increases in $\hat{\mu}_{h}^{(c)}$. for age of children subpopulations are for those aged 25-29 and, especially, 30-39. Moreover, the ratio $\hat{\lambda}$ for children aged 30-39 has decreased from 1.07 to 0.95 . Although the associated confidence interval contains 1 , it is possible that the ratio 0.95 does represent a real discrepancy between $\hat{\mu}_{h}^{(p)}$ and $\hat{\mu}_{h}^{(c)}$. as a result of such an immigration effect. It may be possible to move the ratio closer to 1 by removing immigrants (since 1991) from the 2001 census counts in the poststratification weights, but such a 
modification was not available to us and has not been pursued.

7. Reporting error. In Section 6, we discussed how differences between poststratified estimates $\hat{\mu}^{(p)}$ and $\hat{\mu}^{(c)}$ could depend on the choice of population used to contruct the poststratified weights. In a closely related way, these differences can also depend on the reporting of the variables $m_{i}^{(p)}$ and $m_{i}^{(c)}$ and their subpopulation components.

Consider, for example, the effect of restricting the total population of individuals in GB in 2001 and the analysis sample to those living in private households. To ensure comparability of $\hat{\mu}^{(p)}$ and $\hat{\mu}^{(c)}$, we also need to restrict the measures $m_{i}^{(p)}$ and $m_{i}^{(c)}$ to apply to the counts of non-coresident parents and children living in private households. In fact, it is not possible to apply this restriction to the BHPS variables making up $m_{i}^{(p)}$ and $m_{i}^{(c)}$. Hence, the results cited in the previous section for weights based on the private household population may be viewed as subject to the effect of reporting error because, for example, the reports of non-coresident parents aged $80+$ could be overstated as a result of the inclusion of some parents living in care homes and this could bias $\hat{\mu}_{. k}^{(c)}$ (but not $\hat{\mu}_{\cdot k}^{(p)}$ ) upwards.

A related example concerns the restriction of individuals to those resident in GB. It may be reasonable to assume that the analysis sample and the census population counts obey this restriction. However, there may be cases where the restriction does not hold for the parent or child variables underlying the $m_{i}^{(p)}$ and $m_{i}^{(c)}$. It is possible with the BHPS data to identify whether a parent is deemed by a respondent to live overseas. Restricting non-coresident parents to GB-based ones reduces the number of respondents reporting 1 non-coresident parent by 27 and the number reporting 2 by 71 . This leads to a reduction in $\hat{\mu}^{(c)}$, no effect on $\hat{\mu}^{(p)}$ and increases in $\hat{\lambda}$. For example, the value of 0.9319 reported for $\hat{\lambda}$ in Table 6 for father-daughter dyads is increased to 0.9563 . However, the BHPS data do not permit the same restriction to be applied to reported children. We only know about the child the respondent has most contact with, and we do not know the age or sex of this child. Just over 100 of these children are overseas, but the actual number could be much greater if we assume parents are unlikely to have most contact with a child living overseas (if there are other children still in GB). As we cannot apply the same restriction to the parent and child data, we simply note this potential source of inconsistency between $\hat{\mu}^{(p)}$ and $\hat{\mu}^{(c)}$ and have left the overseas parents in the parent counts used in Section 6.

There are many other possible variables which could be subject to reporting error, not just the examples of residence status (in private household or not) and country of residence (GB or not) discussed above. Given our focus 
on consistency of the parent and child data, we are primarily interested in whether reporting is consistent between these two sources, rather than how this reporting relates to some 'true' variable. For example, in Section 4.1 we discussed the classification of parents or children as biological, adopted or step. The BHPS questions about non-coresident parents make no distinction between these categories and so a broad definition, including all three categories, has to be used for $m_{i}^{(c)}$. We discussed the more complex task of constructing the variable $m_{i}^{(p)}$, number of non-coresident children, and explained why we restricted the definition of this variable to biological children. Given the broader definition of parents, we might anticipate a tendency for $\hat{\mu}^{(c)}$ to exceed $\hat{\mu}^{(p)}$. There is no strong evidence for such an effect in Table 4. It is possible that such an effect is counteracted by the fact that $m_{i}^{(c)}$ is restricted to take a maximum of 2 , that is at most one father and one mother can be reported, whereas it is possible that multiple fathers (biological, adopted or step) or multiple mothers could report the same child and this could inflate $\hat{\mu}^{(p)}$.

One final important source of potential reporting error is the classification of whether a parent or child is coresident or not. For example, the process by which a child leaves a parental home can often take place over a period of time during which the definition of whether the child and its parents are coresident is not clear-cut. It is feasible then that the child and its parents may report this coresidency status differently. The background literature reviewed in Section 2 provides various illustrations of potential reporting error in coresidency status, in particular the tendency for fathers to underreport their non-coresident children, for example by omitting births from their fertility histories, as shown by Rendall et al. (1999). We consider now possible explanations for the two remaining unexplained discrepancies of $\hat{\lambda}$ from 1 in Table 4 in terms of such reporting error.

The first discrepancy is for father-daughter dyads, where $\hat{\lambda}=0.9319$, so that the number of non-coresident daughters reported by men is $7 \%$ lower than would be expected given the number of non-coresident fathers reported by women. If reporting error is the explanation, the results suggest either:

(a) underreporting of non-coresident daughters by men or

(b) overreporting of non-coresident fathers by women (i.e. women are more likely to report having a father than are men).

Let us assume that men underreport their fertility. As $\hat{\lambda}$ for the fatherson dyads is close to 1 , explanation (a) would suggest that fathers are more likely to omit a daughter than a son when reporting the number of noncoresident children. Note, however, that the estimates $\hat{\mu}^{(p)}$ are identical for 
the father-son and father-daughter dyads, while the estimate $\hat{\mu}^{(c)}$ is higher for the father-daughter dyads. If we assume an average sex ratio of 1 among the population with a living father, this would suggest that men are no more likely to underreport daughters than sons, which would favour explanation (b). Perhaps daughters are more likely to stay in contact with their father after parental divorce, and are therefore more likely than sons to report having a non-coresident father.

The second and final discrepancy we consider is for mother-son dyads, where $\hat{\lambda}=1.0774$, so that the number of non-coresident sons reported by women is $8 \%$ higher than would be expected given the number of mothers reported by men. If reporting error is the explanation, the results suggest either:

(a) overreporting of non-coresident sons by women or

(b) underreporting of non-coresident mothers by men.

Comparing patterns in the estimates $\hat{\mu}^{(p)}$ and $\hat{\mu}^{(c)}$ for the mother-son and mother-daughter dyads, we find that $\hat{\mu}^{(p)}$ is slightly higher for the mother-son dyads, but the most striking difference is in $\hat{\mu}^{(c)}$ : the mean number of noncoresident mothers is higher for women than for men. Assuming that mothers correctly report their total (coresident + non-coresident) children, and that children correctly report whether they have a mother, this pattern suggest differential reporting of coresidence, with a greater discrepancy in motherson reports than in mother-daughter reports. Non-coresident daughters are more likely to report being non-coresident than are sons.

8. Estimation of population characteristics. Our comparisons of estimators of a common parameter using parent and child data have so far explored sources of inconsistency between the estimators and ways of reducing inconsistency by refining sample weighting or redefining count variables. In this section, we take the parameter to be of interest for its own sake, not just as a means of assessing data quality. In this case, the choice of sample weighting and variable definition should, of course, depend on the definition of the parameter and this may lead to different choices to those made earlier.

We focus in this section on the question of how to combine the parent and child data to estimate a given parameter of interest $\theta$. Subject to the above remarks about choice of weights, we suppose that, after poststratified weighting, we have estimators $\hat{\theta}^{(p)}$ and $\hat{\theta}^{(c)}$, which are both approximately unbiased for $\theta$ and we consider a combined estimator of $\theta$, given by $\hat{\theta}=\alpha \hat{\theta}^{(p)}+(1-\alpha) \hat{\theta}^{(c)}$, where $\alpha$ is some constant between 0 and 1 . In the supplementary materials we derive the value of $\alpha$ which minimises the 
TABLE 5

Estimates of $\theta_{k}$, the mean number of non-coresident children aged $16+$, for parents in different age groups $k$. Standard errors in parentheses.

\begin{tabular}{|ccccccc|}
\hline Age group $k$ & \multicolumn{2}{c}{$\hat{\theta}_{k}^{(p)}$} & \multicolumn{2}{c}{$\hat{\theta}_{k}^{(c)}$} & \multicolumn{3}{c|}{$\hat{\theta}_{k}^{(\text {opt })}$} \\
\hline $60-69$ & 2.076 & $(0.055)$ & 2.159 & $(0.053)$ & 2.120 & $(0.041)$ \\
$70-79$ & 2.069 & $(0.070)$ & 2.120 & $(0.060)$ & 2.098 & $(0.045)$ \\
$80+$ & 1.964 & $(0.089)$ & 1.754 & $(0.073)$ & 1.843 & $(0.051)$ \\
\hline
\end{tabular}

variance of this estimator and obtain the optimal estimator given by

$\hat{\theta}^{(o p t)}=\hat{\alpha}^{(o p t)} \hat{\theta}^{(p)}+\left(1-\hat{\alpha}^{(o p t)}\right) \hat{\theta}^{(c)}, \quad$ where $\quad \hat{\alpha}^{(o p t)}=\frac{\hat{V}^{(c)}-\hat{C}^{(p c)}}{\hat{V}^{(p)}+\hat{V}^{(c)}-2 \hat{C}^{(p c)}}$

and $\hat{V}^{(p)}, \hat{V}^{(c)}$ and $\hat{C}^{(p c)}$ are estimators of $\operatorname{var}\left(\hat{\theta}^{(p)}\right), \operatorname{var}\left(\hat{\theta}^{(c)}\right)$, and $\operatorname{cov}\left(\hat{\theta}^{(p)}, \hat{\theta}^{(c)}\right)$, respectively. We also show how to estimate the standard error of $\hat{\theta}^{(o p t)}$. To illustrate the potential reduction in standard error achieved by such optimal estimation, we consider a parameter which refers to one of the three subpopulations defined by the parental age groups: 60-69, 70-79 and 80+. Following the notation in Section 3, we denote these subpopulations or age groups by $U_{k}^{(c)}$ or simply by $k$. We define our parameter as the mean number of non-coresident children $A_{i+}$ across parents $i$ in age group $k$, given by $\theta_{k}=\sum_{U_{k}^{(c)}} A_{i+} / N_{k}^{(c)}$, where $N_{k}^{(c)}$ is the size of $U_{k}^{(c)}$. This parameter is a scaled version of the subpopulation mean $\mu_{. k}$ introduced in Section 3 since we have $\theta_{k}=\left(N / N_{k}^{(c)}\right) \mu_{\text {. } k}$.

We shall compare three estimators of $\theta_{k}$ : those based on the parent or child data, denoted respectively by $\hat{\theta}_{k}^{(p)}=\left(N / N_{k}^{(c)}\right) \hat{\mu}_{. k}^{(p)}$ and $\hat{\theta}_{k}^{(c)}=\left(N / N_{k}^{(c)}\right) \hat{\mu}_{. k}^{(c)}$, and the optimal combined estimator, denoted $\hat{\theta}_{k}^{(o p t)}$. In each case we use the poststratified weights described in Section 6 .

Table 5 displays values of these estimators for the three age groups together with estimated standard errors. The optimal estimator is roughly midway between the estimators based on the parent and child data, since $\hat{\alpha}^{(o p t)}$ is not far from 0.5 for each age group $(0.47,0.43$ and 0.42 for ages $60-69,70-79$ and $80+$, respectively). The optimal estimator has considerably reduced standard error for each age group.

In many contexts, it may be of interest to study not only the mean $\theta_{k}$, but also the frequency distribution of the number of non-coresident children, defined as $F_{k}(t)=\sum_{i \in U_{k}^{(c)}} I\left(A_{i+}=t\right)$ for $t=0,1,2, \ldots$ A natural direct 
estimator of $F_{k}(t)$ based upon the parent data is given by

$$
\hat{F}_{k}^{(p)}(t)=(N / n) \sum_{i \in s^{(p)}} w_{P S i}^{(p)} I\left(i \in U_{k}^{(c)}\right) I\left(m_{i}^{(p)}=t\right), \quad t=0,1,2, \ldots
$$

where $w_{P S i}^{(p)}$ is the poststratified weight. We show in the supplementary materials that the sum of these estimated frequencies is $N_{k}^{(c)}$, as we would wish, that the mean of this estimated frequency distribution is $\hat{\theta}_{k}^{(p)}$, as well as how to estimate the standard error of $\hat{F}_{k}^{(p)}(t)$.

We cannot estimate $F_{k}(t)$ directly from the child data. We propose to improve on $\hat{F}_{k}^{(p)}(t)$ instead by calibrating its mean to $\hat{\theta}_{k}^{(o p t)}$ as follows

$$
\hat{F}_{k}^{(r e g)}(t)=\hat{F}_{k}^{(p)}(t)+N_{k}^{(c)} b_{k}(t)\left(\hat{\theta}_{k}^{(o p t)}-\hat{\theta}_{k}^{(p)}\right),
$$

where $b_{k}(t)$ is the estimated regression coefficient of the indicator variable $I\left(m_{i}^{(p)}=t\right)$ on $m_{i}^{(p)}$ :

$$
b_{k}(t)=\frac{\sum_{i \in s^{(p)}} w_{P S i}^{(p)} I\left(i \in U_{k}^{(c)}\right)\left(m_{i}^{(p)}-\bar{m}_{k}\right) I\left(m_{i}^{(p)}=t\right)}{\sum_{i \in s^{(p)}} w_{P S i}^{(p)} I\left(i \in U_{k}^{(c)}\right)\left(m_{i}^{(p)}-\bar{m}_{k}\right)^{2}},
$$

where $\bar{m}_{k}=\sum_{i \in s^{(p)}} w_{P S i}^{(p)} I\left(i \in U_{k}^{(c)}\right) m_{i}^{(p)} / \sum_{i \in s^{(p)}} w_{P S i}^{(p)} I\left(i \in U_{k}^{(c)}\right)$. The estimator $\hat{F}_{k}^{(r e g)}(t)$ may be interpreted as a form of regression estimator (Fuller, 2009). It makes no assumption about the shape of the frequency distribution. We show in the supplementary materials that $\hat{F}_{k}^{(r e g)}(t)$ has sum $N_{k}^{(c)}$ and mean $\hat{\theta}_{k}^{(o p t)}$ as well as how to estimate its standard error.

Estimated frequency distributions using both $\hat{F}_{k}^{(p)}(t)$ and $\hat{F}_{k}^{(r e g)}(t)$ are presented in Table 6 . In Table 5 we saw that the optimal estimate of the mean was greater than the estimate based on the parent data for age groups 60-69 and 70-79. The corresponding increase in the mean of the estimated frequency distributions in Table 6 is achieved by reduced estimated frequencies for 0 and 1 children, increased estimated frequencies for $3+$ children and virtually no change in the estimates for 2 children. The reverse occurs for age group $80+$ where the smaller optimal estimate of the mean in Table 5 corresponds to increased estimated frequencies for 0 and 1 children, a smaller estimated frequency for $3+$ children and again virtually no change in estimate for 2 children. The difference between the standard errors of the two estimators of the frequency distribution in Table 6 depends on the correlation between $I\left(m_{i}^{(p)}=t\right)$ and $m_{i}^{(p)}$. The maximum correlation occurs for $t=3+$ children and this is where the standard error is reduced the most. 
TABLE 6

Estimates of frequencies of numbers of non-coresident children aged $16+$ in thousands and percentages, for parents in different age groups in Great Britain in 2001. Standard errors in parentheses.

\begin{tabular}{|c|c|c|c|c|c|c|}
\hline $\begin{array}{l}\text { Age group } \\
\quad \text { Number of children }\end{array}$ & $\hat{F}_{k}^{(p)}(t)$ & $(\mathrm{SE})$ & $\%$ & $\hat{F}_{k}^{(r e g)}(t)$ & $(\mathrm{SE})$ & $\%$ \\
\hline \multicolumn{7}{|l|}{$60-69$} \\
\hline 0 children & 689 & $(77)$ & 12.9 & 650 & $(69)$ & 12.2 \\
\hline 1 child & 869 & $(82)$ & 16.3 & 844 & (78) & 15.8 \\
\hline 2 children & 1,986 & $(103)$ & 37.2 & 1,982 & $(103)$ & 37.1 \\
\hline $3+$ children & 1,794 & $(106)$ & 33.6 & 1,863 & $(88)$ & 34.9 \\
\hline Total & 5,338 & & 100 & 5,338 & & 100 \\
\hline \multicolumn{7}{|l|}{$70-79$} \\
\hline 0 children & 654 & $(66)$ & 15.6 & 638 & $(60)$ & 15.2 \\
\hline 1 child & 870 & $(78)$ & 20.7 & 858 & $(75)$ & 20.4 \\
\hline 2 children & 1,303 & (89) & 31.0 & 1,302 & $(88)$ & 31.0 \\
\hline $3+$ children & 1,375 & $(90)$ & 32.7 & 1,403 & (68) & 33.4 \\
\hline Total & 4,202 & & 100 & 4,202 & & 100 \\
\hline \multicolumn{7}{|l|}{$80+$} \\
\hline 0 children & 296 & (44) & 12.4 & 330 & $(40)$ & 13.8 \\
\hline 1 child & 676 & (67) & 28.3 & 714 & (61) & 30.0 \\
\hline 2 children & 723 & (63) & 30.3 & 721 & (63) & 30.2 \\
\hline $3+$ children & 690 & (67) & 28.9 & 619 & (53) & 26.0 \\
\hline Total & 2,384 & & 100 & 2,384 & & 100 \\
\hline
\end{tabular}

The correlation is least for $t=2$ children, indeed it is negligible, and in this case the standard error is virtually unchanged. Overall, optimal estimation does offer a decent reduction in standard error despite the fact that no direct estimation of the frequency distribution is feasible using the child data.

9. Concluding remarks. In this paper we have considered a survey design where characteristics of non-coresident dyads in a family network may be estimated using data obtained from either member of each sampled dyad. Thus, in our application with parent-child dyads, the perspective of a survey respondent as either parent or child may be used. We have shown how inconsistencies between estimates obtained from reports from these two perspectives may indicate problems of data quality, specifically selective missingness or reporting error. In our application we found selective missingness provided the most relevant explanation for these inconsistencies and that poststratification was helpful in reducing them. We have also developed methods for combining estimates from parent and child data.

Our focus here has been on the estimation of some fairly simple demo- 
graphic parameters defined in terms of sex and age. This research was originally motivated by similar questions of data quality for a broader range of dyadic variables concerning exchange of support, for example whether a grandparent helps their adult children with childcare. In principle, the methods discussed in this paper could be applied to such questions. For example, the binary variable $A_{i j}$ introduced in Section 3 could be redefined as whether $i$ is a non-coresident parent of $j$ and provides $j$ with help with child care.

The difficulty with applying our methods in this case is that they require data on $A_{i j}$ either at the individual level, for example reports of whether help is provided to each individual non-coresident child, or as a total $\left(A_{i+}\right.$ or $A_{+i}$ in Section 3), for example the number of non-coresident children to which help is provided. The problem is that such data are often not available, because, for example, of the number of questions that would be needed to ask about all children on a range of types of support. The BHPS questions about exchange of support to parents or to children refer collectively to non-coresident children or parents, respectively. Thus, a parent reports whether they help any of their non-coresident children but not how many children. Dealing with 'any' rather than 'how many' children raises significant additional methodological challenges, which we aim to address in further research. Simplistic treatment of 'any' could lead to bias. For example, in a population with many children per family, the proportion of individuals who receive a given form of help from any of their children will typically exceed the proportion of individuals who give this form of help to one of their parents.

Another restriction of the paper has been to estimation of finite population characteristics for descriptive purposes. Many research questions are analytic, however, such as those at the start of Section 1, and may be formulated in terms of models (e.g. Snijders and Kenny, 1999). The relation of the methods in this paper to such modelling is another area for further research.

\section{SUPPLEMENTARY MATERIAL}

\section{Supplementary information.}

(doi: 10.1214/00-AOASXXXXSUPP; .pdf).

\section{REFERENCES}

Altonji, J. G., Hayashi, F. and Kotlikoff, L. J. (1997). Parental altruism and inter vivos transfers: theory and evidence. Journal of Political Economy 105 1121-1166.

Brandt, M., Haberkern, K. and Szydlik, M. (2009). Intergenerational help and care in Europe. European Sociological Review 25 585-601. 
Chan, T. W. and Ermisch, J. (2011). Intergenerational exchange of instrumental support: dynamic evidence from the British Household Survey Working paper, University of Oxford.

Frank, O. (1977). Survey sampling in graphs. Journal of Statistical Planning and Inference 1 235-264.

Fuller, W. A. (2009). Sampling Statistics. Hoboken: Wiley.

Handcock, M. S. and GiLe, K. J. (2007). Modeling social networks with sampled or missing data Working paper 75, Centre for Statistics and the Social Sciences, University of Washington.

HANDCOCK, M. S. and Gile, K. J. (2010). Modeling social networks from sampled data. Annals of Applied Statistics 4 5-25.

Hatton, T. J. (2005). Explaining trends in UK immigration. Journal of Population Economics 18 719-740.

Henretta, J. C., Grundy, E. and Harris, S. (2002). The influence of socio-economic and health differences on parents' provision of help to adult children: a British-United States comparison. Ageing and Society 22 441-458.

ISER (2018). British Household Panel Survey: Waves 1-18, 1991-2009, 8th ed. University of Essex, Institute for Social and Economic Research [original data producer(s)], Colchester, Essex: UK Data Archive [distributor]. SN: 5151.

Lohr, S. L. (2010). Sampling: Design and Analysis, 2nd ed. Boston MA: Brooks/Cole.

Mandemakers, J. J. and Dykstra, P. A. (2008). Discrepancies in parent's and adult child's reports of support and contact. Journal of Marriage and the Family $\mathbf{7 0} 495-506$.

Rendall, M. S. and BALL, D. J. (2004). Immigration, emigration and the ageing of the overseas-born population in the United Kingdom. Population Trends 116 18-27.

Rendall, M. S., Clarke, L., Peters, E. H., Ranjit, N. and Verropoulou, G. (1999). Incomplete reporting of men's fertility in the United States and Britain: a research note. Demography 36 135-144.

Shapiro, A. (2004). Revisiting the generation gap: exploring the relationships of parent/adult-child dyads. International Journal of Aging and Human Development $\mathbf{5 8}$ $127-146$.

Snijders, T. A. B. and Kenny, D. A. (1999). The social relations model for family data: a multilevel approach. Personal Relationships 6 471-486.

Sorenson, E. (1997). A national profile of nonresident fathers and their ability to pay child support. Journal of Marriage and the Family 59 785-808.

Stykes, J. B., Manning, W. D. and Brown, S. L. (2013). Nonresident fathers and formal child support: evidence from the CPS, the NSFG and the SIPP. Demographic Research 29 1299-1330.

Taylor, M. F., Brice, J., Buck, N. and Prentice-Lane, E. (2010). British Household Panel Survey User Manual, Volume A: Introduction, Technical Report and Appendices. University of Essex.

Valliant, R., Dever, J. A. and Kreuter, F. (2013). Practical Tools for Designing and Weighting Survey Samples. Springer, New York.

Widmer, E. D., Aeby, G. and SAPIN, M. (2013). Collecting family network data. International Review of Sociology 23 27-46.

Department of Statistics

London School of Economics \& Political Science

Houghton Street

LONDON WC2A 2AE, UK

E-MAIL: c.j.skinner@lse.ac.uk

f.a.steele@lse.ac.uk 